\title{
छூ \\ Possible superfluidity of molecular hydrogen in a two-dimensional crystal phase of sodium
}

\author{
Claudio Cazorla* \\ Institut de Ciència de Materials de Barcelona (ICMAB-CSIC), 08193 Bellaterra, Spain \\ Jordi Boronat \\ Departament de Física i Enginyeria Nuclear, Universitat Politècnica de Catalunya, Campus Nord B4-B5, E-08034, Barcelona, Spain
}

(Received 30 September 2013; published 3 December 2013)

\begin{abstract}
We theoretically investigate the ground-state properties of a molecular para-hydrogen $\left(\mathrm{p}-\mathrm{H}_{2}\right)$ film in which crystallization is energetically frustrated by embedding sodium $(\mathrm{Na})$ atoms periodically distributed in a triangular lattice. In order to fully deal with the quantum nature of $\mathrm{p}-\mathrm{H}_{2}$ molecules, we employ the diffusion Monte Carlo method and realistic semiempirical pairwise potentials describing the interactions between $\mathrm{H}_{2}-\mathrm{H}_{2}$ and $\mathrm{Na}-\mathrm{H}_{2}$ species. In particular, we calculate the energetic, structural, and superfluid properties of two-dimensional $\mathrm{Na}-\mathrm{H}_{2}$ systems within a narrow density interval around equilibrium at zero temperature. In contrast to previous computational studies considering other alkali metal species such as rubidium and potassium, we find that the $\mathrm{p}-\mathrm{H}_{2}$ ground state is a liquid with a significantly large superfluid fraction of $\rho_{s} / \rho=0.29(2)$. The appearance of $\mathrm{p}-\mathrm{H}_{2}$ superfluid response is due to the fact that the interactions between $\mathrm{Na}$ atoms and $\mathrm{H}_{2}$ molecules are less attractive than between $\mathrm{H}_{2}$ molecules. This induces a considerable reduction of the hydrogen density which favors the stabilization of the liquid phase.
\end{abstract}

DOI: 10.1103/PhysRevB.88.224501

PACS number(s): 67.90.+z, 61.50.Ah, 67.80.-s

\section{INTRODUCTION}

Unlike helium, bulk molecular para-hydrogen $\left(\mathrm{p}-\mathrm{H}_{2}\right)$ always solidifies if a sufficiently low temperature is reached. ${ }^{1}$ Intermolecular $\mathrm{H}_{2}-\mathrm{H}_{2}$ interactions are attractive and quite intense; hence, even though hydrogen molecules are lighter than ${ }^{4} \mathrm{He}$ atoms, $\mathrm{p}-\mathrm{H}_{2}$ crystallization is energetically favored over melting in the $T \rightarrow 0$ limit frustrating any possibility to observe superfluidity (SF) or Bose-Einstein condensation (BEC) in bulk. Putting this into numbers, molecular hydrogen becomes a solid at temperatures below $T_{t} \sim 14 \mathrm{~K}$, whereas the critical temperature at which BEC and SF are expected to occur is $T_{c} \sim 1 \mathrm{~K}^{2}$ In spite of that, many experimental attempts have focused on supercooling bulk liquid $\mathrm{p}-\mathrm{H}_{2}$ below $T_{c}$, although unfortunately with no apparent success to date. ${ }^{3,4}$

A likely way to induce superfluidity in molecular hydrogen consists in lowering its melting temperature by reducing its dimensionality and/or confining it to restricted geometries. Following this line of thinking many experimental and theoretical studies have focused on the characterization and analysis of $\mathrm{p}-\mathrm{H}_{2}$ films adsorbed on different substrates. ${ }^{5-8}$ For instance, two-dimensional hydrogen has been observed to freeze at temperatures around $5 \mathrm{~K}$ when placed onto an exfoliated graphite plate. ${ }^{9}$ Also, it has been experimentally shown that small para-hydrogen clusters immersed in ${ }^{4} \mathrm{He}$ droplets exhibit superfluidlike behavior. ${ }^{10}$ On the theoretical side, it has been predicted that one-dimensional arrays of $\mathrm{p}-\mathrm{H}_{2}$ molecules remain in the liquid phase down to absolute zero ${ }^{11}$ and that small two- and three-dimensional clusters of pure $\mathrm{p}-\mathrm{H}_{2}$ are superfluid at temperatures below $1-2 \mathrm{~K} .^{12,13}$

An alternative way to induce superfluidity in molecular para-hydrogen may consist in embedding alkali metal (AM) atoms on it. This idea was originally proposed by Gordillo and Ceperley (GC) ${ }^{14}$ and is based on the fact that the interactions between alkali metal atoms and molecular hydrogen are less attractive than between $\mathrm{p}-\mathrm{H}_{2}$ molecules. Therefore, a substantial reduction of the equilibrium hydrogen density can be induced which triggers stabilization of the liquid. In particular, GC investigated two-dimensional $\mathrm{AM}-\mathrm{H}_{2}(\mathrm{AM}=\mathrm{K}$ and $\mathrm{Cs}$ ) systems at low temperatures (i.e., 1-4 K) employing the path integral Monte Carlo (PIMC) technique. They found that the $\mathrm{p}-\mathrm{H}_{2}$ equilibrium state in $\mathrm{AM}-\mathrm{H}_{2}$ films was a liquid of concentration $\sim 0.04 \AA^{-2}$ which became superfluid at temperatures below 1.2 K. Nevertheless, a few years later Boninsegni $^{15}$ found, using a very similar approach to GC and attempting extrapolation to the thermodynamic limit, that the hydrogen equilibrium state in $\mathrm{K}-\mathrm{H}_{2}$ films was a crystal commensurate with the underlying lattice of alkali metal atoms. The superfluid fraction of such a commensurate system was equal to zero as reported by Boninsegni. Almost simultaneously to the publication of Boninsegni's work, ${ }^{15}$ Cazorla and Boronat presented a ground-state study (i.e., performed at zero temperature) of a two-dimensional system composed of $\mathrm{Rb}$ atoms and hydrogen molecules. ${ }^{16}$ By using the diffusion Monte Carlo (DMC) method and somewhat more realistic $\mathrm{AM}-\mathrm{H}_{2}$ potentials than adopted by $\mathrm{GC}$ and Boninsegni, they found that the $\mathrm{p}-\mathrm{H}_{2}$ ground state in the $\mathrm{Rb}-\mathrm{H}_{2}$ film was a highly structured liquid with a practically suppressed superfluid fraction of $\rho_{s} / \rho=0.08(2)$. Overall, these theoretical predictions appeared to suggest that the embedding of alkali metal atoms on hydrogen matrices was not an effective strategy to trigger $\mathrm{p}-\mathrm{H}_{2}$ superfluidity.

In this work we report an exhaustive diffusion Monte Carlo (DMC) study of the ground-state properties (i.e., energetic, structural, and superfluid) of $\mathrm{p}-\mathrm{H}_{2}$ molecules within a twodimensional solid matrix of sodium $(\mathrm{Na})$ atoms. Our main finding is that the $\mathrm{p}-\mathrm{H}_{2}$ ground state is a liquid that possesses a remarkably large superfluid fraction of $\rho_{s} / \rho=0.29(2)$. The reason behind such a large superfluid response lies in the details of the $\mathrm{Na}-\mathrm{H}_{2}$ interaction, which presents a smaller repulsive core as compared to other $\mathrm{AM}-\mathrm{H}_{2}$ pairwise potentials.

The organization of this article is as follows. In the next section we provide a brief description of the DMC method and the details of our calculations. Next, we present our results and 
compare them with previous computational works. Finally, we summarize our main findings in Sec. IV.

\section{METHODS AND SIMULATION DETAILS}

The basics of the diffusion Monte Carlo (DMC) method have been reviewed with detail elsewhere ${ }^{17-20}$ so here we only comment on the essential ideas.

In the DMC approach, the time-dependent Schrödinger equation of a quantum system of $N$ interacting particles is solved stochastically by simulating the time evolution of the Green's function propagator $e^{-\frac{i}{\hbar} \hat{H} t}$ in imaginary time $\tau \equiv \frac{i t}{\hbar}$. For $\tau \rightarrow \infty$, sets of configurations (walkers) $\left\{\mathbf{R}_{i} \equiv\right.$ $\left.\mathbf{r}_{1}, \ldots, \mathbf{r}_{N}\right\}$ rendering the probability distribution function $\left(\Psi_{0} \Psi\right)$ are generated, where $\Psi_{0}$ is the ground-state wave function of the system and $\Psi$ is a guiding wave function (gwf) used for importance sampling. Within DMC, exact results (i.e., subject only to statistical uncertainties) are obtained for the total ground-state energy and related quantities in bosonic quantum systems. ${ }^{21-23}$

We are interested in studying the ground state of a system of $\mathrm{p}-\mathrm{H}_{2}$ molecules immersed in a two-dimensional solid matrix of $\mathrm{Na}$ atoms. We model the Hamiltonian of this system as

$$
H=-\frac{\hbar^{2}}{2 m_{\mathrm{H}_{2}}} \sum_{i=1}^{N} \nabla_{i}^{2}+\sum_{i<j}^{N} V_{\mathrm{H}_{2}-\mathrm{H}_{2}}\left(r_{i j}\right)+\sum_{i, k}^{N, n} V_{\mathrm{Na}^{-} \mathrm{H}_{2}}\left(R_{i k}\right),
$$

where $m_{\mathrm{H}_{2}}$ is the mass of a p- $\mathrm{H}_{2}$ molecule, $N$ is the number of hydrogen molecules, $n$ is the number of alkali metal atoms, and $V_{\mathrm{H}_{2}-\mathrm{H}_{2}}$ and $V_{\mathrm{N} a-\mathrm{H}_{2}}$ are semiempirical pairwise potentials describing the $\mathrm{H}_{2}-\mathrm{H}_{2}$ and $\mathrm{Na}-\mathrm{H}_{2}$ interactions. The internal structure of $\mathrm{p}-\mathrm{H}_{2}$ molecules has been neglected (i.e., vibrational and rotational degrees of freedom are disregarded) and the hydrogen-hydrogen molecular interactions have been modeled with the standard Silvera-Goldman potential. ${ }^{24}$ The interactions between $\mathrm{Na}$ atoms and $\mathrm{H}_{2}$ molecules are described with a Lennard-Jones potential of the form $V_{\mathrm{LJ}}(r)=$ $4 \epsilon\left[(\sigma / r)^{12}-(\sigma / r)^{6}\right]$, with parameters taken from Ref. 25, namely $\sigma=4.14 \AA$ and $\epsilon=30 \mathrm{~K}$. The kinetic energy of the $\mathrm{Na}$ atoms has been also neglected since this is expected to be much smaller than the typical energy scale of $\mathrm{p}-\mathrm{H}_{2}$ molecules (i.e., $10-100 \mathrm{~K}$ ).

It is worth noticing that despite asymptotic DMC results do not depend on the choice of the guiding wave function (gwf), the algorithmic efficiency in DMC runs is influenced by the quality of $\Psi$. The guiding wave function that we use to describe the present $\mathrm{Na}-\mathrm{H}_{2}$ system contains correlations between the $N$ $\mathrm{H}_{2}$ molecules $\left[\mathrm{f}_{2}\left(r_{i j}\right)\right]$ and the $N \mathrm{H}_{2}$ molecules and $n$ alkali metal atoms $\left[\mathrm{F}_{2}\left(R_{i j}\right)\right]$. In the liquid phase this gwf reads

$$
\Psi_{L}\left(\mathbf{r}_{1}, \mathbf{r}_{2}, \ldots, \mathbf{r}_{N}\right)=\prod_{i<j}^{N} \mathrm{f}_{2}\left(r_{i j}\right) \prod_{i, k}^{N, n} \mathrm{~F}_{2}\left(R_{i k}\right)
$$

where two-body correlation factors $\mathrm{f}_{2}(r)$ and $\mathrm{F}_{2}(r)$ have been chosen of the McMillan form, i.e., $\exp \left[-\frac{1}{2}(b / r)^{5}\right]$, and $R_{i k}$ is the distance between the $i$ th $\mathrm{p}-\mathrm{H}_{2}$ molecule and the $k$ th alkali atom. In order to compute the energy of possible solid pseudocommensurate phases (see next section for details), we adopted the guiding wave function

$$
\Psi_{S}\left(\mathbf{r}_{1}, \mathbf{r}_{2}, \ldots, \mathbf{r}_{N}\right)=\Psi_{L} \prod_{i=1}^{N} g_{1}\left(\xi_{i}\right)
$$

where one-body factors $g_{1}$ are Gaussians, i.e., $\exp \left(-\frac{1}{2} c r^{2}\right)$, and $\xi_{i}$ is the distance of the $i$ th $\mathrm{p}-\mathrm{H}_{2}$ molecule to its site in the corresponding perfect lattice configuration. The value of all variational parameters were determined through subsidiary variational Monte Carlo calculations. In the liquid phase these resulted in $b=3.70 \AA\left(\mathrm{f}_{2}\right)$ and $5.60 \AA\left(\mathrm{F}_{2}\right)$, and in the solid phase, $b=3.45 \AA\left(\mathrm{f}_{2}\right), 5.60 \AA\left(\mathrm{F}_{2}\right)$, and $c=1.22 \AA^{-2}\left(\mathrm{~g}_{1}\right)$.

In our simulations, both the alkali metal atoms and $\mathrm{p}-\mathrm{H}_{2}$ molecules are arranged in a strictly two-dimensional geometry. $\mathrm{Na}$ atoms are considered static and distributed according to a triangular lattice of parameter $10 \AA$. It is worth noticing that such an alkali metal geometry is realistic since it has been experimentally observed in $\mathrm{Ag}(111)$ plates. $^{26-28}$ In order to determine the equation of state and ground-state properties of the liquid $\mathrm{H}_{2}$ film, we kept the number of alkali metal atoms fixed to 30 and progressively increased the concentration of $\mathrm{p}-\mathrm{H}_{2}$ molecules. The typical size of our simulation boxes is $50 \AA \times 50 \AA$. The value of the technical parameters in the calculations were set to ensure convergence of the total energy per particle to less than $0.1 \mathrm{~K} /$ atom. For instance, the mean population of walkers was equal to 400 and the length of the imaginary time step $(\Delta \tau)$ to $5 \times 10^{-4} \mathrm{~K}^{-1}$. Statistics were accumulated over $10^{5} \mathrm{DMC}$ steps performed after equilibration of the system and the approximation used for the short-time Green's function $e^{-\hat{H} \tau}$ is exact up to order $(\Delta \tau)^{2} \cdot{ }^{20,29}$ It is important to stress that by using the same DMC method we have been able to reproduce in previous works the experimental equation of state of archetypal quantum solids like ${ }^{4} \mathrm{He}, \mathrm{H}_{2}$, $\mathrm{LiH}$, and $\mathrm{Ne} .{ }^{30-38}$

\section{RESULTS AND DISCUSSION}

Let us to start by presenting the energy results obtained in the liquid $\mathrm{Na}-\mathrm{H}_{2}$ system. The corresponding total, potential, and kinetic energies per hydrogen molecule expressed as a function of density are enclosed in Table I (potential energies were obtained with the pure estimator technique, hence all the reported energies are exact, i.e., subject to statistical uncertainty only $\left.{ }^{22,23}\right)$. The ground-state energy and equilibrium density of the liquid film $e_{0}$ and $\rho_{0}$ were determined by fitting

TABLE I. Total $(E)$, potential $(V)$, and kinetic $(T)$ energies per $\mathrm{p}-\mathrm{H}_{2}$ molecule calculated in the liquid $\mathrm{H}_{2}$ system and expressed in units of $\mathrm{K}$.

\begin{tabular}{lccc}
\hline \hline$\rho\left(\AA^{-2}\right)$ & $E / N$ & $\langle V\rangle / N$ & $\langle T\rangle / N$ \\
\hline 0.029 & $-45.04(4)$ & $-63.65(7)$ & $18.61(7)$ \\
0.031 & $-45.66(3)$ & $-65.52(5)$ & $19.86(5)$ \\
0.033 & $-46.24(2)$ & $-67.02(5)$ & $20.78(5)$ \\
0.036 & $-46.93(2)$ & $-69.36(7)$ & $22.43(7)$ \\
0.038 & $-47.13(2)$ & $-71.57(7)$ & $24.44(7)$ \\
0.040 & $-46.86(3)$ & $-72.95(5)$ & $26.09(5)$ \\
\hline \hline
\end{tabular}




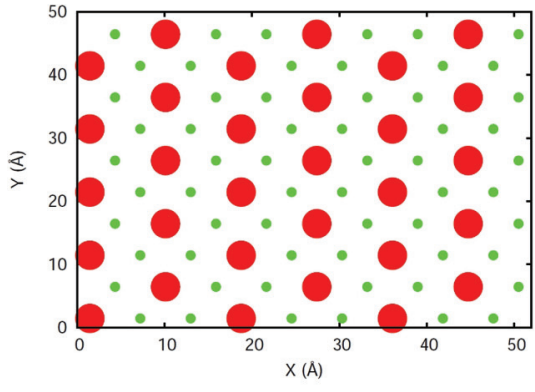

$C^{\prime} 1 / 3$

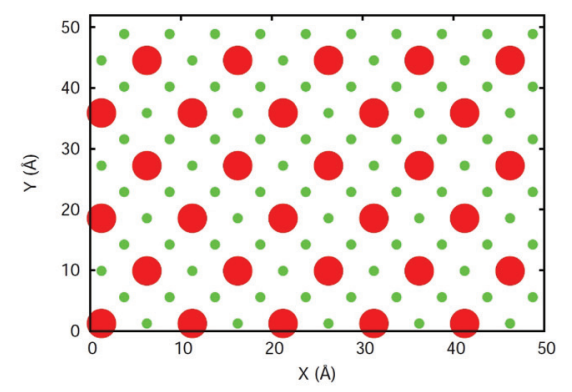

$C^{\prime} 1 / 4$

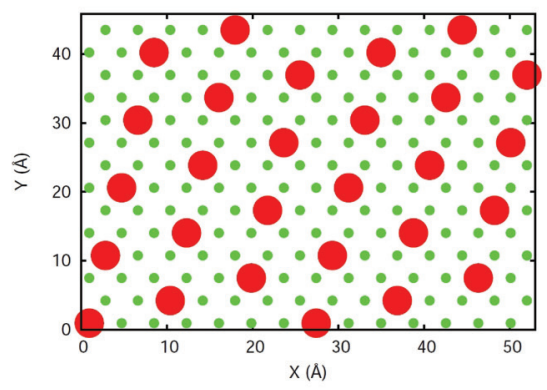

$C^{\prime} 1 / 7$

FIG. 1. (Color online) Representation of the three pseudocommensurate crystal structures considered in this work. Big red dots represent sodium atoms and small green dots $\mathrm{p}-\mathrm{H}_{2}$ molecules.

the polynomial curve

$$
e(\rho)=e_{0}+B\left(\frac{\rho-\rho_{0}}{\rho_{0}}\right)^{2}+C\left(\frac{\rho-\rho_{0}}{\rho_{0}}\right)^{3}
$$

to the calculated total energies. The resulting optimal parameter values are $B=86.16 \mathrm{~K}, C=221.69 \mathrm{~K}, \rho_{0}=0.038 \AA^{-2}$, and $e_{0}=-47.13 \mathrm{~K}$. We note that the liquid ground-state energy and equilibrium density are significantly different from those computed in the pure two-dimensional $\mathrm{p}-\mathrm{H}_{2}$ crystal, namely $-23.41 \mathrm{~K}$ and $0.067 \AA^{-2} .^{30}$ These large total energy and equilibrium density differences have their origin in the potential energy gain and steric effects deriving from the presence of sodium atoms. Also, we observe that the equilibrium density of the liquid $\mathrm{H}_{2}$ film is appreciably larger than the calculated in the analogous $\mathrm{Rb}-\mathrm{H}_{2}$ system, namely $0.023 \AA^{-2}$ (Ref. 16) (e.g., the ratio among the number of $\mathrm{p}-\mathrm{H}_{2}$ molecules and alkali metal atoms are $10 / 3$ and $2 / 1$, respectively). The cause for the large equilibrium density in the $\mathrm{Na}$ case, as compared to that of the $\mathrm{Rb}$ system, is related to the decrease of the core size of the AM- $\mathrm{H}_{2}$ interaction (i.e., $\sigma=4.54 \AA$ in the $\mathrm{Rb}-\mathrm{H}_{2}$ case), which makes the surface available to $\mathrm{p}-\mathrm{H}_{2}$ molecules larger (we note that the depth of the potential wells $\epsilon$ in both $\mathrm{Na}-\mathrm{H}_{2}$ and $\mathrm{Rb}-\mathrm{H}_{2}$ interactions are very similar, i.e., 30 and $28 \mathrm{~K}$, respectively ${ }^{25}$ ).

Regarding the stabilization of possible pseudocommensurate solid phases, we investigated the three crystal structures shown in Fig. 1 We refer to them as pseudocommensurate phases because in order to fully fulfill commensurability some $\mathrm{p}-\mathrm{H}_{2}$ molecules should be located at the same $x-y$ positions as alkali metal atoms. Since the system considered in the present study is strictly two dimensional, this positional coincidence is energetically forbidden. Therefore, we started by generating the exact $C_{1 / 3}, C_{1 / 4}$, and $C_{1 / 7}$ commensurate structures (where the subscripts indicate the relative population of alkali and hydrogen species) and then removed by hand the $\mathrm{p}-\mathrm{H}_{2}$ molecules located at the same positions as sodium atoms (hence the prime in our notation). In Table II we enclose the energy results obtained for those pseudocommensurate structures. It is found that of the three cases considered $C_{1 / 4}^{\prime}$ is by far the system with the lowest energy. Interestingly, the density of the $C_{1 / 4}^{\prime}$ phase (i.e., $0.035 \AA^{-2}$ ) is very close to the equilibrium density found in the analogous liquid system (i.e., $0.038 \AA^{-2}$ ). Moreover, from a structural point of view the pseudocommensurate $C_{1 / 4}^{\prime}$ phase is very similar to the equilibrium state predicted by Boninsegni in $\mathrm{K}-\mathrm{H}_{2}$ films at low temperatures (see Fig. 3 in Ref. 15). Nevertheless, the total energy per particle of the $C_{1 / 4}^{\prime}$ phase is about $0.9 \mathrm{~K}$ larger than the energy of the corresponding fluid at equilibrium and thus, according to our calculations, the ground state of the $\mathrm{Na}-\mathrm{H}_{2}$ system is a liquid. In view of these findings, we will concentrate on the description of the liquid $\mathrm{H}_{2}$ system in the remainder of this article.

In Fig. 2 we show a snapshot of the probability density calculated for the ground state of the $\mathrm{Na}-\mathrm{H}_{2}$ system. There it is observed that hydrogen molecules can access a large portion of the surface left between $\mathrm{Na}$ atoms by diffusing through honeycomblike pathways created around the alkali metal centers. This situation is eminently different from the one observed in $\mathrm{Rb}-\mathrm{H}_{2}$ films, where a highly structured liquid is found to be the ground state (see Ref. 16 and Fig. 2). In this last case, most of $\mathrm{p}-\mathrm{H}_{2}$ molecules are localized within the interior of the triangles formed by $\mathrm{Rb}$ atoms and the connectivity between high-density $\mathrm{p}-\mathrm{H}_{2}$ regions is rather low. The probability density differences observed between $\mathrm{Na}-\mathrm{H}_{2}$ and $\mathrm{Rb}-\mathrm{H}_{2}$ systems again can be understood in terms of the core lengths of the corresponding $\mathrm{AM}-\mathrm{H}_{2}$ interactions.

The enhanced delocalization of $\mathrm{p}-\mathrm{H}_{2}$ molecules in the Nabased film can be also deduced from the shape of the pairradial distribution functions calculated with the pure estimator technique. ${ }^{22,23}$ In Fig. 3 one can observe that the peaks of the crossed $g_{\mathrm{Na}-\mathrm{H}_{2}}$ distribution function are less sharp than those obtained in the analogous $\mathrm{Rb}-\mathrm{H}_{2}$ system $[g(r)$ results from Ref. 16 have been included in the plot for comparison purposes]. Also, the first peak of the $g_{\mathrm{H}_{2}-\mathrm{H}_{2}}$ function centered at $r=3.9 \AA$ is a global maximum and does not coincide with the position of the first $g_{\mathrm{Na}-\mathrm{H}_{2}}$ peak found at $r=5.1 \AA$, so implying a high concentration of $\mathrm{p}-\mathrm{H}_{2}$ molecules. These last features are in opposition to what is observed in the $\mathrm{Rb}-\mathrm{H}_{2}$ film, where the first and second $g_{\mathrm{H}_{2}-\mathrm{H}_{2}}$ peaks centered at 5.2 and $10.3 \AA$ can be ascribed to the hexagonal-like pattern that

TABLE II. Total $(E)$, potential $(V)$, and kinetic $(T)$ energies per p$\mathrm{H}_{2}$ molecule calculated in three different pseudocommensurate solid $\mathrm{H}_{2}$ systems and expressed in units of $\mathrm{K}$.

\begin{tabular}{lcrcc}
\hline \hline Phase & $\rho\left(\AA^{-2}\right)$ & $E / N$ & $\langle V\rangle / N$ & $\langle T\rangle / N$ \\
\hline$C_{1 / 3}^{\prime}$ & 0.023 & $-41.56(2)$ & $-53.74(3)$ & $12.18(3)$ \\
$C_{1 / 4}^{\prime}$ & 0.035 & $-46.28(2)$ & $-67.76(5)$ & $21.48(5)$ \\
$C_{1 / 7}^{\prime}$ & 0.069 & $58.47(2)$ & $-22.11(4)$ & $80.58(4)$ \\
\hline \hline
\end{tabular}



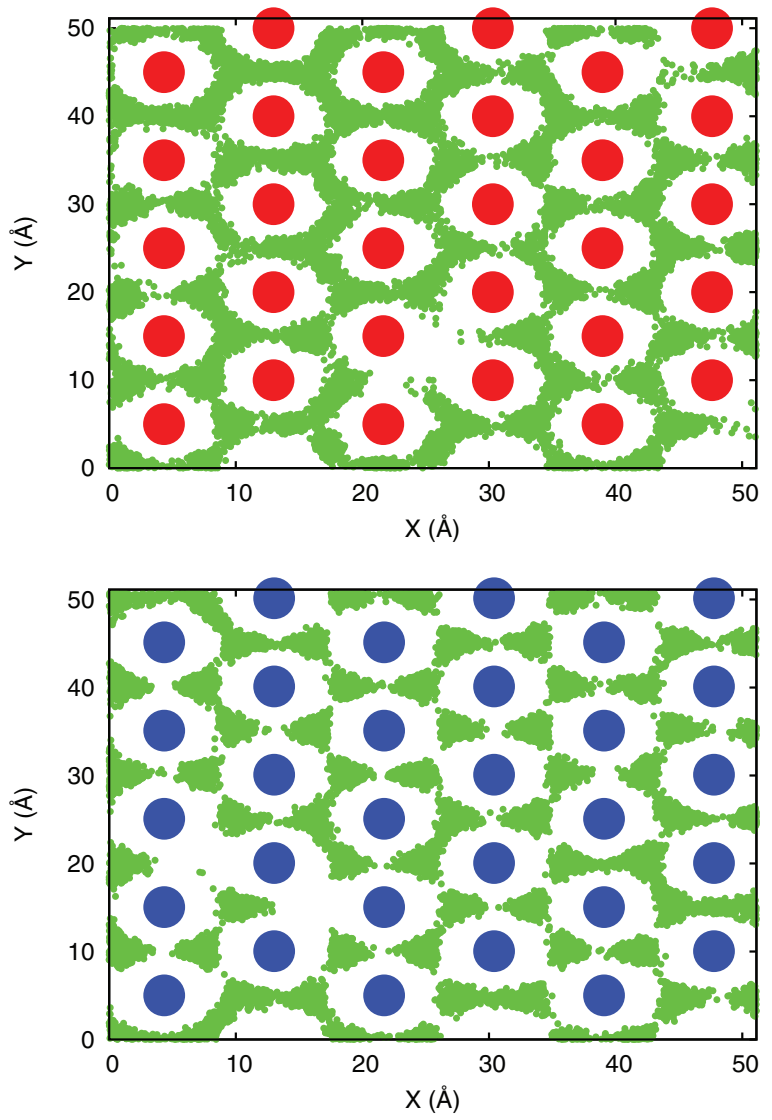

FIG. 2. (Color online) Snapshot of the probability density of twodimensional $\mathrm{p}-\mathrm{H}_{2}$ (green dots) calculated in the liquid $\mathrm{Na}-\mathrm{H}_{2}(\mathrm{Na}=$ red dots, top $)$ and $\mathrm{Rb}-\mathrm{H}_{2}(\mathrm{Rb}=$ blue dots, bottom) films at equilibrium (i.e., 0.038 and $0.023 \AA^{-2}$, respectively) and zero temperature.

results from filling the triangles formed by $\mathrm{Rb}$ atoms with one $\mathrm{p}-\mathrm{H}_{2}$ molecule.

In order to better assess the structure of the $\mathrm{p}-\mathrm{H}_{2}$ molecules in the $\mathrm{Na}$ film, we calculated the corresponding structure factor $S(\mathbf{k})$ using the pure estimator technique ${ }^{22,23}$ (see Fig. 4). This has been obtained through estimation of the expected value of the density fluctuation operator $\frac{1}{\sqrt{N}} \sum_{i}^{N} \exp \left(-i \mathbf{k} \cdot \mathbf{r}_{\mathbf{i}}\right)$, multiplied by its conjugate. Quantities $g_{\mathrm{H}_{2}-\mathrm{H}_{2}}$ and $S(\mathbf{k})$ are obviously related by a Fourier transform. In Fig. 4 one can observe the presence of two sharp $S(\mathbf{k})$ peaks centered at reciprocal lattice vectors that essentially coincide with the periodicity imposed by the triangular $\mathrm{Na}$ lattice (i.e., at $\mathbf{k}=$ 0.75 and $1.5 \AA^{-1}$ ). However, no other large scattering peaks signalizing the appearance of a solid or glassy state are seen in the figure. The $\mathrm{p}-\mathrm{H}_{2}$ film, therefore, appears to be a fluid. We note that moderate structure factor fluctuations around unity appear in the regime of large k's; these are probably due to the large $\mathrm{H}_{2}-\mathrm{H}_{2}$ distance variations that hydrogen molecules may experience when they are close to an alkali metal center.

As an additional test, we also monitored the average distance that the $\mathrm{p}-\mathrm{H}_{2}$ molecules move away from the $\mathrm{Na}$ atoms which at the start of the simulation are closest to them. We plot this quantity as a function of imaginary time in Fig. 5. As it can be appreciated, function $\Delta r(\tau)=\left\langle\left|\mathbf{r}_{i}(\tau)-\mathbf{R}_{n i}(0)\right|\right\rangle$ monotonically increases with $\tau$ reproducing so the typical profile that is obtained in bulk fluids (i.e., is roughly linear).
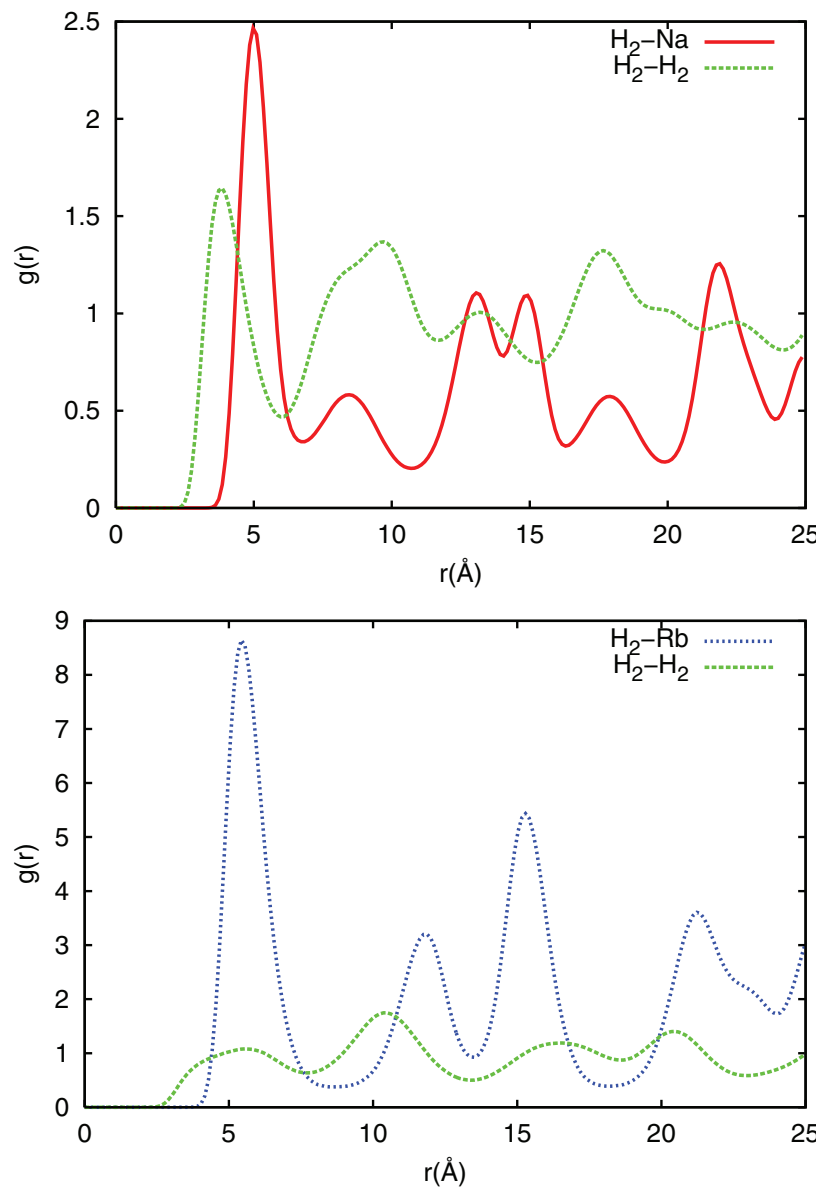

FIG. 3. (Color online) Pair-radial distribution functions (pure estimation) obtained in the liquid $\mathrm{Na}-\mathrm{H}_{2}$ (top) and $\mathrm{Rb}-\mathrm{H}_{2}$ (bottom) films at their corresponding equilibrium densities.

Assuredly, then, the simulated $\mathrm{p}-\mathrm{H}_{2}$ system remains in a liquid phase. For comparison purposes, we include also in Fig. 5 the diffusion profile obtained in the equivalent $\mathrm{Rb}-\mathrm{H}_{2}$ system under equilibrium conditions. In this last case, the mobility of

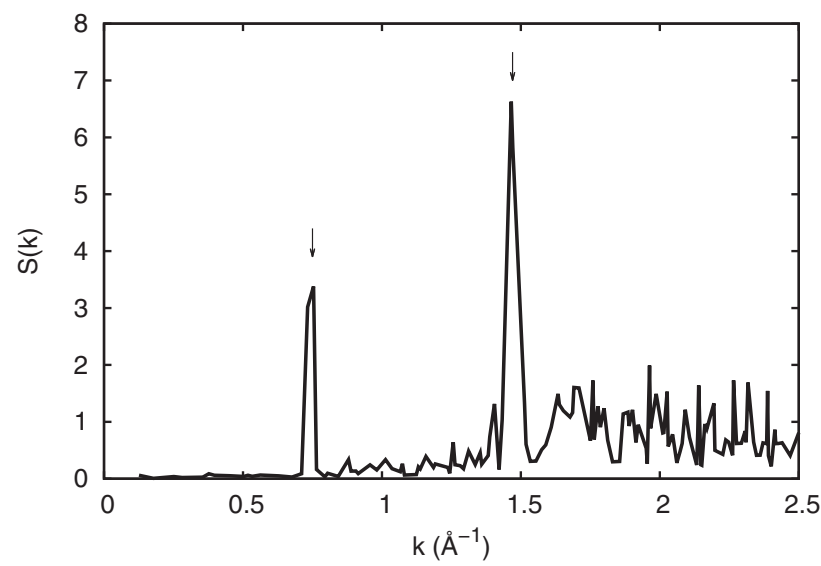

FIG. 4. Molecular hydrogen structure factor (pure estimation) obtained in the liquid $\mathrm{Na}-\mathrm{H}_{2}$ system at the equilibrium density. Peaks ascribed to the periodicity of the underlying sodium film are indicated with arrows. 


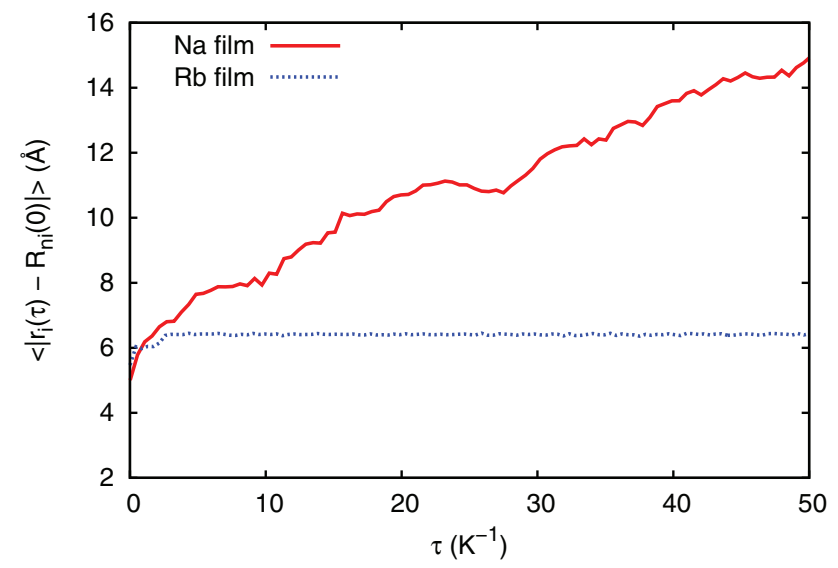

FIG. 5. (Color online) Averaged mean distance of $\mathrm{p}-\mathrm{H}_{2}$ molecules to the nearest $\mathrm{Na}$ and $\mathrm{Rb}$ atoms at the start of the simulations carried out at the respective equilibrium densities.

the hydrogen molecules is practically suppressed as shown by the computed $d \Delta r / d \tau \sim 0$ slope.

In order to complete our description of the $\mathrm{Na}-\mathrm{H}_{2}$ film, we estimated the superfluid fraction $\rho_{s} / \rho$ of the $\mathrm{p}-\mathrm{H}_{2}$ subsystem. This can be achieved within the DMC formalism by extending to zero temperature the winding number technique employed in PIMC calculations. ${ }^{39,40}$ This generalization essentially consists of reinterpreting the winding number in terms of the trajectory of particles in DMC simulations subject to periodic boundary conditions. In particular, $\rho_{s} / \rho$ is computed as the ratio of two diffusion constants $D_{s} / D_{0}$ in the imaginary time limit $\tau \rightarrow \infty$, where $D_{0}=\hbar^{2} /\left(2 m_{\mathrm{H}_{2}}\right)$ and

$$
D_{s}=\lim _{\tau \rightarrow \infty} \frac{N}{4 \tau} \frac{\int d \mathbf{R} f(\mathbf{R}, \tau)\left[\mathbf{R}_{\mathrm{c} . \mathrm{m} .}(\tau)-\mathbf{R}_{\mathrm{c} . \mathrm{m} .}(0)\right]^{2}}{\int d \mathbf{R} f(\mathbf{R}, \tau)} .
$$

In Eq. (5), $\mathbf{R}_{\text {c.m. }}=(1 / N) \sum_{i=1}^{N} \mathbf{r}_{i}$ represents the position of the $\mathrm{p}-\mathrm{H}_{2}$ center of mass, $f(\mathbf{R}, \tau)$ is the probability distribution $\Psi_{0} \Psi$, and the factor of 4 in the denominator corresponds to two times the dimensionality. In Fig. 6 we plot the $D_{s} / D_{0}$ function computed at two different densities and expressed as a function of imaginary time. From the $D_{s} / D_{0}$ asymptote we estimate the superfluid fraction of the hydrogen liquid

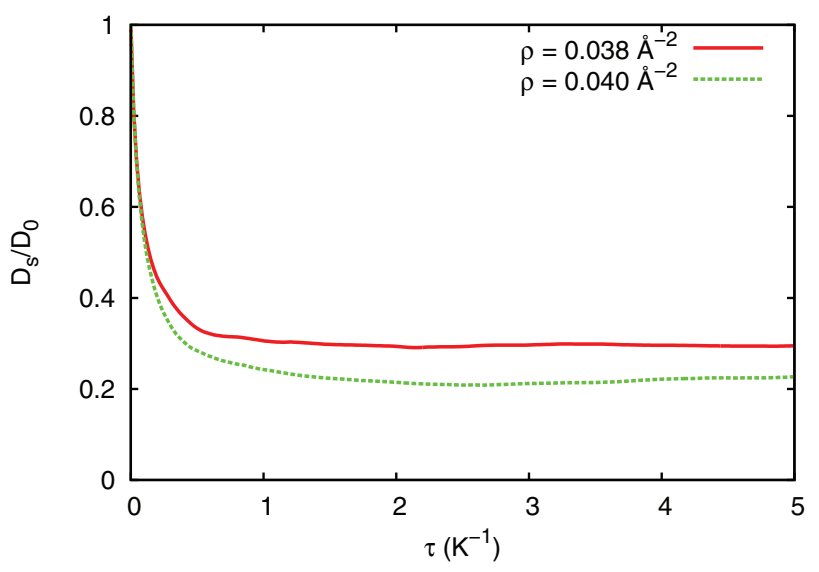

FIG. 6. (Color online) Diffusion Monte Carlo estimation of the $\mathrm{p}-\mathrm{H}_{2}$ superfluid density in $\mathrm{Na}$ films at different densities. at equilibrium to be $0.29(2)$, a quite large value. It is worth recalling that the superfluid fraction computed in the equivalent $\mathrm{Rb}-\mathrm{H}_{2}$ system was much smaller, namely $\rho_{s} / \rho=0.08(2) .{ }^{16}$ This significant $\rho_{s} / \rho$ difference is a direct consequence of the increase in the $\mathrm{p}-\mathrm{H}_{2}$ concentration at equilibrium, which in turn depends on the form of the AM- $\mathrm{H}_{2}$ interaction. Furthermore, we calculated the superfluid response of the $\mathrm{Na}-\mathrm{H}_{2}$ film at a density slightly above the equilibrium point (see Fig. 6) and found that the value of the $D_{s} / D_{0}$ asymptote decreases [i.e., $0.23(2)$ at $\rho=0.040 \AA^{-2}$ ]. This last finding points out to a strong dependence of the superfluid fraction on $\rho$ due to the effect of excluding surface produced by the presence of static $\mathrm{Na}$ atoms. We note that the finite size of the simulation box could induce some dependence of the superfluid fraction on the number of particles. In order to reduce this effect, however, we worked with a rather large simulation box of typical size $50 \AA \times 50 \AA$ (i.e., as large as the one employed in Ref. 15, where suppression of $\mathrm{p}-\mathrm{H}_{2}$ superfluidity in a potassium film was predicted). Also the effect of adding a third dimension in the direction that is perpendicular to the alkali metal plane could change our $\rho_{s} / \rho$ results. However, based on previous outcomes obtained in pure two- and quasi-two-dimensional ${ }^{4} \mathrm{He}$ systems, ${ }^{34}$ we foresee that considering this effect, within the typical values observed in adsorbed monolayers, will further enhance superfluidity.

In the light of our energy, structural, and superfluid fraction results obtained in alkali- $\mathrm{H}_{2}$ films (see Ref. 16) it may be concluded that (i) the softer the repulsive core of the crossed AM- $\mathrm{H}_{2}$ interaction is, the larger the $\mathrm{p}-\mathrm{H}_{2}$ equilibrium density and superfluid fraction result, and (ii) the superfluid response of two-dimensional p- $\mathrm{H}_{2}$ films strongly depends on density.

\section{CONCLUSIONS}

Summarizing, we have performed an exhaustive diffusion Monte Carlo study of the energetic, structural, and superfluid properties of a $\mathrm{p}-\mathrm{H}_{2}$ two-dimensional system in which $\mathrm{Na}$ atoms have been embedded forming a triangular lattice. The main motivation of this computational study was to investigate whether hydrogen crystallization could be prevented in two dimensions and, if so, to estimate the superfluid response of the corresponding $\mathrm{p}-\mathrm{H}_{2}$ subsystem at zero temperature. We have found, in contrast to previous computational works considering other alkali metal species and $\mathrm{AM}-\mathrm{H}_{2}$ potentials, that the $\mathrm{p}-\mathrm{H}_{2}$ ground state in the $\mathrm{Na}$ film is a liquid that possesses a remarkably large superfluid fraction [i.e., $\rho_{s} / \rho=0.29(2)$ ]. The principal reason behind the stabilization of this fluid relies on the fact that $\mathrm{Na}-\mathrm{H}_{2}$ interactions are less attractive than $\mathrm{H}_{2}-\mathrm{H}_{2}$ and thus a significant reduction of the hydrogen equilibrium density occurs. Also, we have found that the energetic, structural, and superfluid properties of $\mathrm{p}-\mathrm{H}_{2}$ films strongly depend on density.

Importantly, we note that small variations of the crossed AM- $\mathrm{H}_{2}$ potential parameters may lead to appreciable differences on the computed $\mathrm{p}-\mathrm{H}_{2}$ properties. Therefore, since there are few potentials in the literature which describe the interactions between alkali metal atoms and $\mathrm{p}-\mathrm{H}_{2}$ molecules accurately, and those which have been reported probably are not too versatile, we must be cautious with our conclusions. More realistic and transferable alkali- $\mathrm{H}_{2}$ potentials than 
currently available are urgently needed to provide decisive hints in the quest for realizing $\mathrm{p}-\mathrm{H}_{2}$ superfluidity. Nevertheless, in view of the great fundamental interest of possible $\mathrm{p}-\mathrm{H}_{2}$ superfluidity, we strongly encourage experimental realizations of molecular hydrogen films adsorbed on $\mathrm{Na}$ substrates.

\section{ACKNOWLEDGMENTS}

This work was supported by MICINN-Spain (Grants No. MAT2010-18113, No. CSD2007-00041, and No. FIS201125275), Generalitat de Catalunya (Grant No. 2009SGR-1003), and the CSIC JAE-doc (C.C.) program. *ccazorla@icmab.es

${ }^{1}$ J. van Straaten, R. J. Wijngaarden, and I. F. Silvera, Phys. Rev. Lett. 48, 97 (1982).

${ }^{2}$ O. N. Osychenko, R. Rota, and J. Boronat, Phys. Rev. B 85, 224513 (2012).

${ }^{3}$ G. M. Seidel, H. J. Maris, F. I. B. Williams, and J. G. Cardon, Phys. Rev. Lett. 56, 2380 (1986).

${ }^{4}$ H. J. Maris, G. M. Seidel, and F. I. B. Williams, Phys. Rev. B 36, 6799 (1987).

${ }^{5}$ J. De Kinder, A. Bouwen, and D. Schoemaker, Phys. Rev. B 52, 15782 (1995).

${ }^{6}$ D. F. Brewer, J. C. N. Rajendra, and A. L. Thomson, J. Low Temp. Phys. 101, 317 (1995).

${ }^{7}$ M. Schindler, A. Dertinger, Y. Kondo, and F. Pobell, Phys. Rev. B 53, 11451 (1996).

${ }^{8}$ P. E. Sokol, R. T. Azuah, M. R. Gibbs, and S. M. Bennington, J. Low Temp. Phys. 103, 23 (1996).

${ }^{9}$ F. C. Liu, Y. M. Liu, and O. E. Vilches, Phys. Rev. B 51, 2848 (1995).

${ }^{10}$ S. Grevenev, B. Sartakov, J. P. Toennius, and A. F. Vilesov, Science 289, 1532 (2000).

${ }^{11}$ M. C. Gordillo, J. Boronat, and J. Casulleras, Phys. Rev. Lett. 85, 2348 (2000).

${ }^{12}$ M. C. Gordillo and D. M. Ceperley, Phys. Rev. B 65, 174527 (2002).

${ }^{13}$ P. Sindzingre, D. M. Ceperley, and M. L. Klein, Phys. Rev. Lett. 67, 1871 (1991).

${ }^{14}$ M. C. Gordillo and D. M. Ceperley, Phys. Rev. Lett. 79, 3010 (1997).

${ }^{15}$ M. Boninsegni, New J. Phys. 7, 78 (2005).

${ }^{16}$ C. Cazorla and J. Boronat, J. Low. Temp. Phys. 134, 43 (2004).

${ }^{17}$ B. L. Hammond, W. A. Lester, Jr., and P. J. Reynolds, in Monte Carlo Methods in Ab Initio Quantum Chemistry (World Scientific, Singapore, 1994).

${ }^{18}$ R. Guardiola, Lect. Notes Phys. 510, 269 (1998).
${ }^{19}$ D. M. Ceperley and M. H. Kalos, in Monte Carlo Methods in Statistics Physics (Springer, Berlin, 1986).

${ }^{20}$ J. Boronat and J. Casulleras, Phys. Rev. B 49, 8920 (1994).

${ }^{21}$ By related quantities is meant the expected value of operators $\hat{A}$ that commute with the Hamiltonian, namely $[\hat{A}, \hat{H}]=0$. It is also possible to obtain virtually exact results for $[\hat{A}, \hat{H}] \neq 0$ operators by using forward walking based techniques (see Refs. 22 and 23).

${ }^{22}$ R. Barnett, P. Reynolds, and W. A. Lester Jr., J. Comput. Phys. 96, 258 (1991).

${ }^{23}$ J. Casulleras and J. Boronat, Phys. Rev. B 52, 3654 (1995).

${ }^{24}$ I. F. Silvera and V. V. Goldman, J. Chem. Phys. 69, 4209 (1978).

${ }^{25}$ F. Ancilotto, E. Cheng, M. W. Cole, and F. Toigo, Z. Phys. B 98, 323 (1995).

${ }^{26}$ G. S. Leatherman and R. D. Diehl, Phys. Rev. B 53, 4939 (1996).

${ }^{27}$ R. D. Diehl (private communication).

${ }^{28}$ Gerald S. Leatherman, Ph.D. thesis, Penn State University, 1996.

${ }^{29}$ S. A. Chin, Phys. Rev. A 42, 6991 (1990).

${ }^{30}$ C. Cazorla and J. Boronat, Phys. Rev. B 78, 134509 (2008).

${ }^{31}$ C. Cazorla and J. Boronat, J. Phys.: Condens. Matter 20, 015223 (2008).

${ }^{32}$ J. Boronat, C. Cazorla, D. Colognesi, and M. Zoppi, Phys. Rev. B 69, 174302 (2004).

${ }^{33}$ C. Cazorla and J. Boronat, Phys. Rev. B 77, 024310 (2008).

${ }^{34}$ C. Cazorla, G. Astrakharchick, J. Casulleras, and J. Boronat, J. Phys.: Condens. Matter 22, 165402 (2010).

${ }^{35}$ C. Cazorla, G. Astrakharchick, J. Casulleras, and J. Boronat, New J. Phys. 11, 013047 (2009).

${ }^{36}$ M. C. Gordillo, C. Cazorla, and J. Boronat, Phys. Rev. B 83, 121406(R) (2011).

${ }^{37}$ Y. Lutsyshyn, C. Cazorla, G. E. Astrakharchik, and J. Boronat, Phys. Rev. B 82, 180506(R) (2010).

${ }^{38}$ C. Cazorla and J. Boronat, J. Low Temp. Phys. 139, 645 (2005).

${ }^{39}$ S. Zhang, N. Kawashima, J. Carlson, and J. E. Gubernatis, Phys. Rev. Lett. 74, 1500 (1995).

${ }^{40}$ E. L. Pollock and D. M. Ceperley, Phys. Rev. B 36, 8343 (1987). 